\title{
Corrigendum
}

\section{Dietary glycaemic index and glycaemic load among Australians children and adolescents - results from the 2011-2012 Australian Health Survey - Corrigendum}

\author{
Molly E. Jones, Alan W. Barclay, Jennie C. Brand-Miller and Jimmy Chun Yu Louie \\ (First published online 18 August 2016)
}

DOI: 10.1017/S0007114516001823. Published by Cambridge University Press, 12 May 2016.

There were errors in the original text of the article - please see below for both the original texts and the corrections.

Original text

Based on this method, 244 extreme low reporters and 530 extreme high reporters were identified. A sensitivity analysis was performed where results of all participants and only those with plausible data were compared, and no material differences in the direction of trends and conclusions were observed. Hence the extreme mis-reporters were excluded in the final analysis. The final dataset included 1876 individuals, of which $51 \cdot 3 \%$ were boys.

Correction

Based on this method, 248 extreme low reporters and 82 extreme high reporters were identified. Another 457 respondents were excluded as they did not have their weight recorded, which disallowed the computation of the EI:BMR ratio for the Goldberg cut-off assessment. A sensitivity analysis was performed where results of all participants and only those with plausible data were compared, and no material differences in the direction of trends and conclusions were observed. Furthermore, in view of the considerable number of persons excluded based on the fact that data on body weight were not available, additional sensitivity analyses were performed including persons for whom it was not possible to judge whether they had over-or underreported their dietary intake $(\mathrm{n}=457)$. These analyses yielded findings similar to those reported for the sample on which the main analyses were based (weighted $\mathrm{n}=1876$ ) (data not shown), i.e. conclusions were not affected by excluding persons without body weight data. Hence the extreme mis-reporters and participants without body weight data were excluded in the final analysis. The final weighted dataset included 1876 individuals, of which 51.3\% were boys.

Original text

Extreme low-reporters excluded from the analysis were older $(12.7 \pm 4.6$ years $)$ and had a higher mean $\mathrm{BMI}$ of $22.5 \pm 4.7 \mathrm{~kg} / \mathrm{m}^{2}$; whereas extreme high-reporters excluded had a slightly lower mean age of $9 \cdot 1 \pm 4 \cdot 8$ years, and a lower $\mathrm{BMI}$ of $17 \cdot 6 \pm 3 \cdot 2 \mathrm{~kg} / \mathrm{m}^{2}$, compared with participants with plausible dietary intake (all $p<0.001$ tested by ANOVA).

Correction

Extreme low-reporters excluded from the analysis were older $\left(12.6 \pm 4.6\right.$ years) and had a higher mean $\mathrm{BMI}$ of $22.5 \pm 4.7 \mathrm{~kg} / \mathrm{m}^{2}$; whereas extreme high-reporters excluded had a slightly lower mean age of $8 \cdot 0 \pm 4.9$ years, and a lower $\mathrm{BMI}$ of $17 \cdot 6 \pm 3 \cdot 2 \mathrm{~kg} / \mathrm{m}^{2}$, compared with participants with plausible dietary intake (all $p<0 \cdot 001$ tested by ANOVA).

The authors apologise for these errors.

\section{Reference}

Jones M, Barclay AW, Brand-Miller JC, et al. (2016) Dietary glycaemic index and glycaemic load among Australian children and adolescents: results from the 2011-2012 Australian Health Survey. Br J Nutr 116, 178-187. doi:10.1017/S0007114516001823. 